\title{
Application of the Envelope Peaks over Threshold (EPOT) Method for Probabilistic Assessment of Dynamic Stability
}

\author{
Bradley Campbel ${ }^{1}$, Vadim Belenky ${ }^{1}$, Vladas Pipiras ${ }^{2}$ \\ ${ }^{1}$ David Taylor Model Basin - NSWCCD, 9500 Macarthur Blvd., W. Bethesda, MD, USA. \\ ${ }^{2}$ University of North Carolina at Chapel Hill, 305 Hanes Hall, Chapel Hill, NC USA
}

\begin{abstract}
This paper reviews the research and development of the Envelope Peaks over Threshold (EPOT) method that has taken place in the last three years. The EPOT method is intended for the statistical extrapolation of ship motions and accelerations from time-domain numerical simulations, or possibly, from a model test. To model the relationship between probability and time, the large roll angle events must be independent, so Poisson flow can be used. The method uses the envelope of the signal to ensure the independence of large exceedances. The most significant development was application of the Generalized Pareto Distribution (GPD) for approximation of the tail, replacing the previously used Weibull distribution. This paper reviews the main aspects of modeling the GPD, including its mathematical justification, fitting the parameters of the distribution, and evaluating the probability of exceedance and its confidence interval.
\end{abstract}

Keywords: Large roll, Statistical extrapolation, Generalized Pareto Distribution, EPOT

\section{INTRODUDCTION}

The rarity of dynamic stability failures in realistic sea condition makes the problem of extrapolation inevitable. This can be illustrated in the following example. If we assume an hourly stability failure rate of $10^{-6} \mathrm{hr}^{-1}$ (Kobylinski and Kastner, 2003), then we can expect to see (on average) one failure every 1,000,000 hours. If we require 10 observations for a reliable statistical estimate; then we need to simulate 10,000,000 hours. Even if an advanced hydrodynamic code could run in a real time and a cluster with 1,000 processors is dedicated to the task, it would take 10,000 hours per condition (combination of seaway, speed and heading) to perform the assessment. The cost of the calculations prohibits direct simulation in this manner.

Additionally stability failure is associated with large-amplitude motions and is expected to be nonlinear. Indeed, capsize is related to the ultimate nonlinearity - transition to another equilibrium. In order to have enough fidelity to model this problem, the hydrodynamic code must be quite sophisticated (see a review by Reed, et al., 2014). The probability of capsizing is the topic of a multi-year ONR research project titled "A Probabilistic Procedure for Evaluating the Dynamic Stability and Capsizing of Naval Vessels" (Belenky, et al., 2015). 
IMO document SLF 54/3/1 (Annex 1) defines intact stability failure as a state of inability of a ship to remain within design limits of roll (heel, list) angle combined with high rigid body accelerations. This includes also partial stability failure when a ship is subjected to a large roll angle or excessive accelerations, but does not capsize. Following the same logic one could also include an excessive pitch angle. As this study focuses on partial stability failure, peak over threshold method (POT) was chosen (Pickands, 1975). Introducing a threshold allows considering the data that are more influenced by nonlinearity; this incorporates changing physics into the statistical estimates.

To satisfy the requirement of independent peaks over threshold, the peaks of envelope were used instead of the peaks of the process itself (Campbell and Belenky, 2010). The review of this research effort is available from Belenky and Campbell (2012). That work included consideration of the relationship between probability and time, the probabilistic properties of peaks, application of envelope theory and the extreme value distribution.

The relationship between time and probability is key to the proper treatment of the partial stability failures. It may be modeled with Poisson, which requires the independence of the failure events. In the case of capsizing, the enforcement of Poisson Flow is not required, since capsizing can only occur once per record (the possibility of several capsizings within one record can be safely ignored for practical cases). Belenky and Campbell (2012) also review different ways of statistical characterization of the rate of events, the only parameter of the Poisson flow.

Classical POT methods use the Generalized Pareto Distribution (GPD) to approximate the tail of the distribution above a threshold. However, under certain conditions the GPD may be right bounded, that is, there is some value above which the probability of exceedances is zero. This is not a problem for conventional statistical consideration, when we are interested in the quantiles of the GPD (i.e. the probability is given and the level needs to be found). In ship stability generally the failure level is known and related to down flooding or cargo shifting angles and probability is to be found. The physical meaning of the right bound was not clear at that time (and still is not completely clear). As a result, the Weibull distribution was used for modeling the tail.

Normally distributed wave elevation was the subject of study in Belenky and Campbell (2012). This was a logical first test for these techniques. The study concluded that the distribution of large absolute values of peaks can be approximated by Rayleigh law. The Rayleigh distribution is a particular case of Weibull distribution when the shape parameter equals two. Thus, deviation of this parameter from two may be suitable for representing nonlinearity in a dynamical system.

To investigate the performance of a POT scheme based on the Weibull distribution, a model representing ship motions with realistic stability variation was used (Weems and Wundrow, 2013; Weems and Belenky, 2015). It was found that Weibull distribution does not have enough flexibility to approximate the tail of large-amplitude ship motions and the consideration of the GPD was started again. 
Application of the GPD with EPOT produced very reasonable results (Smith and Zuzick, 2015). The techniques used to fit GPD, estimate the probability of exceedance of a given level and evaluate its uncertainty are described in Campbell, et al. (2014, 2014a) and Glotzer, et al. (2016) and briefly reviewed in the rest of this paper.

\section{MATHEMATICAL BACKGROUND}

\subsection{Distribution of Order Statistics}

In order to understand why statistical extrapolation is possible when the underlying distribution is unknown, we begin with order statistics.

Consider a set of $n$ independent realizations of random variable $z$. Assume that the distribution is given in a form of a cumulative distribution function (CDF) and probability density function (PDF). Sorting the observed values from the largest to smallest we have:

$$
y_{i}=\operatorname{sort}\left(z_{i}\right) \quad i=1, \ldots n
$$

Indeed, for randomly selected values $y$ and $z$ :

$$
p d f(y)=p d f(z) ; \quad C D F(y)=C D F(z)
$$

Consider a value that happens to be $k$-th in the list $(1 \leq k \leq n)$. It is a random number, because, if one generates another set of realizations of variable $z$, and sorts them, another value will be the $k$ th. This random number is referred as $k$-th order statistic. Like any other random variable, $y_{k}$ has its own distribution. This distribution is (see, e.g. David and Nagaraja, 2003):

$$
p d f(y \mid k)=p d f(y) \frac{n !}{(k-1) !(n-k) !} \cdot(C D F(y))^{k-1}(1-C D F(y))^{n-k}
$$

\subsection{Generalized Extreme Value (GEV) Distribution}

Consideration of distribution of the largest value $(k=1)$ when the number of observations $n$ grows, leads to a limit, known as Generalized Extreme Value (GEV) distribution (see e.g. Coles, 2001):

$$
p d f(x)=\frac{1}{\sigma}\left(1+\xi \frac{x-\mu}{\sigma}\right)^{-\left(1+\frac{1}{\xi}\right)} \cdot \exp \left(-\left(1+\xi \frac{x-\mu}{\sigma}\right)^{\frac{1}{\xi}}\right)
$$

$\xi$ is a shape parameter, $\sigma$ is scale parameter $(\sigma>0) ; \mu$ is a shift parameter, Equation (4) is nonzero for: 


$$
\begin{aligned}
& x>\mu-\frac{\sigma}{\xi} \quad \text { for } \quad \xi>0 \\
& x<\mu-\frac{\sigma}{\xi} \quad \text { for } \quad \xi<0
\end{aligned}
$$

and is zero otherwise. If the shape parameter $\xi=0$ :

$$
p d f(x)=\frac{1}{\sigma} \exp \left(\frac{x-\mu}{\sigma}\right) \cdot \exp \left(-\exp \left(\frac{x-\mu}{\sigma}\right)\right)
$$

for any values of $x$.

It is important that the limit (4-6) does not depend on the distribution $z$. That means that all the extreme values have the same distribution if one considers a sample of sufficient volume. This is the essence of the extreme value theorem, sometimes referred to as the Fisher-TippetGnedenko theorem (see, e.g. Coles, 2001).

Direct application of the extreme value theorem for probabilistic assessment of dynamic stability can be found in MacTaggart, (2000), MacTaggart and deKat (2000). However, several issues remained unresolved; including the question how large the sample should be (in terms of record length and number of records) to claim limiting properties of GEV.

\subsection{Generalized Pareto Distribution (GPD)}

The large sample volume needed for direct application of the GEV is partially driven by the fact that only a single value (the largest one from the time window) is used to find the parameters of distribution. The desire to use more data leads to the idea of peaks over threshold methods.

Take $\mu$ as a threshold and find the distribution of the data exceeding this threshold, i.e. consider conditional probability. The Generalized Pareto distribution is derived from the GEV with the threshold condition applied. The basic logic of this derivation is available in Coles (2001). The GPD is expressed as

$$
f(x)=\left\{\begin{array}{c}
\frac{1}{\sigma}\left(1+\xi \frac{x-\mu}{\sigma}\right)^{-\left(1+\frac{1}{\xi}\right)} ; \\
\text { if } \quad \mu<x, \quad \xi>0, \quad \text { or } \\
\mu<x<\mu-\frac{\sigma}{\xi}, \quad \xi<0 \\
\frac{1}{\sigma} \exp \left(-\frac{x-\mu}{\sigma}\right) ; \\
\text { if } \quad \mu<x, \quad \xi=0
\end{array}\right.
$$

where $\xi$ is the shape parameter, $\sigma$ is the scale parameter $(\sigma>0)$ and $\mu$ is the threshold, above which, the GPD is believed to be applicable. Zero-value of the shape parameter is an important 
particular case, approximating the tail of some practically important distributions, including normal and Rayleigh distributions.

Equation (7) expresses the second extreme value theorem, referred as Pickands-Balkema-de Haan theorem. It states that the tail of independent random variables can be approximated with the GPD.

\section{FITTING THE GPD}

\subsection{Preparing Independent Data}

Ship motions are characterized by strong dependence of the data points on each other, especially in following and stern quartering seas when a spectrum is narrow and autocorrelation function takes a long time to decay. However, the peaks of the piecewise linear envelope (see Figure 1) represent independent data points. The difference between piecewise linear and theoretical envelope is considered by Belenky and Campbell (2012). However, this technique may not work for the cases of parametric roll, where mutual dependence is much stronger. The method of collecting independent data for the case of parametric roll is described in Kim, et al. ( 2014).

\subsection{Estimating Shape and Scale Parameters}

To facilitate the choice of the threshold at a later step, fitting the shape and scale parameters are carried out for a series of prescribed thresholds. The maximum likelihood estimator (MLE) method is a standard way of estimating the parameters for the GPD. The idea of MLE method is quite intuitive: to find such values of parameters that are "most likely" to fit the data.

What is "most likely"? The data points that have been observed are the facts. At the same time they are instances of a random variable. Because these particular values were observed, they are more likely to occur than others. That means that the probability of observing these particular values reaches maximum when the correct parameters are used for distribution. The parameters are found by maximizing the value of the likelihood function. In practice this is made easier by taking the natural logarithm of the likelihood function, as the likelihoods usually are small values; also products are transformed into sums, providing additional convenience (Equation 8 below).

$$
\begin{aligned}
& l(\xi, \sigma)=-n \cdot \ln (\sigma)-\left(1+\frac{1}{\xi}\right) \cdot \sum_{i=1}^{n} \ln \left(1+\xi \cdot \frac{z_{i}}{\sigma}\right) \\
& z_{i}=x_{i}-\mu
\end{aligned}
$$

Where $n$ is the number of data points above a threshold $\mu, z_{i}$ are the sample data points above a threshold $\mu$ (sometimes referred to as excesses). 


\subsection{Distribution the GPD Parameters}

Since the shape and scale parameters are estimated from the envelope peak data (which are random numbers), the estimated parameters are also random numbers. Their distribution can be approximated with a bivariate normal distribution (Smith, 1987).

$$
f_{N}(\hat{\xi}, \hat{\sigma})=\frac{1}{2 \pi V_{\xi} V_{\sigma} \sqrt{1-\rho_{\xi \sigma}^{2}}} \cdot \exp \left(-\frac{1}{2\left(1-\rho_{\xi \sigma}^{2}\right)} \cdot\left(\frac{\left(\hat{\xi}-E_{\xi}\right)^{2}}{V_{\xi}}+\frac{\left(\hat{\sigma}-E_{\sigma}\right)^{2}}{V_{\sigma}}-\frac{2 \rho_{\xi \sigma}\left(\hat{\xi}-E_{\xi}\right)\left(\hat{\sigma}-E_{\sigma}\right)}{\sqrt{V_{\xi} V_{\sigma}}}\right)\right)
$$

Here $\hat{\xi}$ and $\hat{\sigma}$ are estimates of $\xi$ and $\sigma, E$ is used for expected (or mean) value, $V$ is the variance and $\rho$ is the correlation coefficient between $\xi$ and $\sigma$. The parameter estimates produced by maximizing equation (8) are the mean values, while the covariance matrix $M_{C}$ is found using the method outlined below.

$$
M_{C}=\left(\begin{array}{cc}
V_{\xi} & \rho_{\xi \sigma} \sqrt{V_{\xi} V_{\sigma}} \\
\rho_{\xi \sigma} \sqrt{V_{\xi} V_{\sigma}} & V_{\sigma}
\end{array}\right)
$$

The delta method allows one to find the estimates of mean and variance of the output, if estimates of the input are known and the function that turns input into output can be linearized, such as by a Taylor series. Because of this the delta method is an approximation. Appling the delta method to maximization of equation (8) yields the Fisher information matrix $M_{F}$ that is an inverse of the covariance matrix (10) (Boos and Stefanski, 2013):

$$
M_{F}=\left(\begin{array}{cc}
-\frac{\partial^{2} l(\xi, \sigma)}{\partial \xi^{2}} & -\frac{\partial^{2} l(\xi, \sigma)}{\partial \xi \partial \xi \sigma} \\
-\frac{\partial^{2} l(\xi, \sigma)}{\partial \xi \partial \xi \sigma} & -\frac{\partial^{2} l(\xi, \sigma)}{\partial \sigma^{2}}
\end{array}\right)
$$

The derivatives in (11) are expressed as follows:

$$
\begin{aligned}
& \frac{\partial^{2} l(\xi, \sigma)}{\partial \xi^{2}}=\frac{2}{\xi^{3}} \sum_{i=1}^{n} \ln \left(1+\xi \cdot \frac{z_{i}}{\sigma}\right)-\frac{2}{\xi^{2}} \sum_{i=1}^{n} \frac{z_{i}}{\sigma+\xi \cdot z_{i}}-\left(1+\frac{1}{\xi}\right) \cdot \sum_{i=1}^{n} \frac{z_{i}^{2}}{\left(\sigma+\xi \cdot z_{i}\right)^{2}} \\
& \frac{\partial^{2} l(\xi, \sigma)}{\partial \sigma^{2}}=\frac{n}{\xi \cdot \sigma^{2}}-\left(1+\frac{1}{\xi}\right) \cdot \sum_{i=1}^{n} \frac{1}{\left(\sigma+\xi \cdot z_{i}\right)^{2}}(13) \\
& \frac{\partial^{2} l(\xi, \sigma)}{\partial \xi \partial \sigma}=\frac{n}{\xi^{2} \cdot \sigma}-\frac{1}{\xi^{2}} \sum_{i=1}^{n} \frac{1}{\sigma+\xi \cdot z_{i}}-\left(1+\frac{1}{\xi}\right) \cdot \sum_{i=1}^{n} \frac{z_{i}}{\left(\sigma+\xi \cdot z_{i}\right)^{2}}
\end{aligned}
$$

The covariance matrix is finally found as:

$$
M_{C}=M_{F}^{-1}
$$

Equations (10) through (14) completely define the bivariate normal distribution (9), as shown in Figure 2. 
It is important to note that the scale parameter is by definition a positive quantity, while the bivariate normal distribution formally supports negative values for the scale parameter. To avoid negativity of $\sigma$ as an artifact of approximation (9), Glotzer, et al. (2016) proposed using the $\hat{l}_{\sigma}=\ln \hat{\sigma}$ instead of $\sigma$. This leads to a new distribution:

$$
\begin{aligned}
& f_{L N}\left(\hat{\xi}, \hat{l}_{\sigma}\right)=\frac{1}{2 \pi V_{\xi} V_{l \sigma} \sqrt{1-\rho_{\xi l \sigma}^{2}}} \cdot \exp \left(-\frac{1}{2\left(1-\rho_{\xi l \sigma}^{2}\right)} \cdot\left(\frac{\left(\hat{\xi}-E_{\xi}\right)^{2}}{V_{\xi}}+\frac{\left(\hat{l}_{\sigma}-E_{l \sigma}\right)^{2}}{V_{l \sigma}}-\frac{2 \rho_{\xi l \sigma}\left(\hat{\xi}-E_{\xi}\right)\left(\hat{l}_{\sigma}-E_{l \sigma}\right)}{\sqrt{V_{\xi} V_{l \sigma}}}\right)\right) \\
& E_{l \sigma}=\ln \left(E_{\sigma}\right) ; \quad V_{l \sigma}=\frac{V_{\sigma}}{E_{\sigma}^{2}} ; \quad \rho_{\xi l \sigma}=\frac{\rho_{\xi \sigma}}{E_{\sigma}} \quad(17)
\end{aligned}
$$

Difference between distribution (16) and bivariate normal (9) is not very large (Glotzer, et al., 2016).

\subsection{Choice of the Threshold}

Choosing a correct threshold is critical to ensuring the applicability of the GPD. If the threshold is too low, the fitted GPD is not an approximation of the tail, because the conditions of the second extreme value theorem have not been met. If the threshold is too high, "eligible" data have been wasted and the result will be more scatter or uncertainty than necessary.

The second extreme value theorem states that the GPD can be used for approximation of the tail of any distribution if the threshold is high enough. That means that above a certain threshold the GPD approximation must be invariant to the threshold (Coles, 2001). The simplest way is to observe stabilization of the shape parameter, see Figure 3.

Campbell, et al. (2014) describes the study of five different methods of setting the threshold:

1. Stabilization of shape parameter

2. Stabilization of modified scale parameter

3. Stabilization of mean residual life estimate

4. Ad-hoc method based on minimum absolute difference between the shape parameter and its median above the threshold

5. Ad-hoc method based on minimum squared difference in the shape from its mean above the threshold

The first three methods are taken from Coles (2001) and they were mostly intended for "manual" calculation with "a human in a loop". The methods 4 and 5 are similar to the methods proposed in Reiss and Thomas (2007) for automatic choice of the threshold. The referred study (Campbell, et al., 2014) has shown that automation of the method 1 through 3 makes the threshold lower than the visual choice. For the example shown in Figure 3, these methods put the threshold somewhere around 13 14 degrees, while the visual choice is somewhere above 17 degrees. 
At the same time methods 4 and 5 have returned the threshold that is more close to the "visual" choice. The method 4 is quickly reviewed below. It is based on minimizing the following function:

$$
f\left(\mu_{k}\right)=\frac{1}{N_{T r}-1-k} \sum_{i=k}^{N_{T r}-1}\left(N_{T r}-i\right)^{b}\left|\hat{\xi}_{i}-\operatorname{median}\left(\hat{\xi}_{k}, . ., \hat{\xi}_{N_{T r}}\right)\right|
$$

The value of $b$ was taken as $0.5 ; N_{T r}$ is the number of the thresholds considered. A plot of (18) is shown in Figure 4. A global minimum (ignoring that the function goes to zero at the right) occurs just below $\mu=17.4 \mathrm{deg}$, which is close to one of the visual choices made at Figure 3.

\section{EXTRAPOLATION AND UNCERTAINTY OF THE PROBABILITY OF EXCEEDANCE}

\subsection{Extrapolated Estimate}

Using the GPD to extrapolate the probability of exceedance yields the conditional probability that the level of interest $c$ has been exceeded if the threshold $\mu$ has been exceeded:

$$
\hat{P}=\left\{\begin{array}{lll}
\left(1+\hat{\xi} \cdot \frac{c-\mu}{\hat{\sigma}}\right)^{-\frac{1}{\hat{\xi}}} & \text { if } \quad \hat{\xi}>-\frac{\hat{\sigma}}{c-\mu} \\
0 & \text { if } \quad \hat{\xi} \leq-\frac{\hat{\sigma}}{c-\mu}
\end{array}\right.
$$

$\mathrm{c}$ is the limit that constitutes the stability failure. The probability that $\mu$ has been exceeded can be estimated statistically, since it has been exceeded often enough so that we have enough data to build the distribution of peaks above it.

Now let's consider the problem of the right bound. As can be seen from equation (19), the probability of exceedance is equal zero for negative shape parameters and $c \geq \mu-\hat{\sigma} / \hat{\xi}$. This has several implications.

First, the extrapolated estimate is a random number. The joint distribution of the shape and scale parameters is approximated using distribution (16). That also means that the mean values of the shape and scale parameters are the most probable values at the same time (because normal distribution has a maximum at its mean value). Thus, one could expect that the most probable values used for formulae (19) returns the most probable value of extrapolated estimate.

So if the formula (19) returns zero, it is the most probable answer, but not the only one possible. In fact, the formula (19) must be accompanied with confidence interval that may be seen as a "range of answers". 


\subsection{Mean and Distribution of the Extrapolated Estimate}

In contrast to the most probable value of the extrapolated estimate, the mean value is never zero for a finite volume of data. Consider formula (19) as a deterministic function of random arguments:

$$
\hat{P}=g(\hat{\xi}, \hat{\sigma})
$$

Its mean value can be found as using well known formula for the mean value of deterministic function of random arguments:

$$
E(\hat{P})=\int_{0}^{\infty} \int_{-\infty}^{\infty} g(\xi, \sigma) f_{L N}(\xi, \sigma) d \xi d \sigma
$$

Using (21) the PDF of the extrapolated estimate is derived as:

$$
f_{p}(x)=\int_{-\infty}^{\infty} f_{L N}\left(\xi, \frac{\xi(c-\mu)}{x^{-\xi}-1}\right) \cdot \frac{\xi^{2} x^{-\xi-1}}{\left(x^{-\xi}-1\right)^{2}}(c-\mu) d \xi
$$

Figure 5 depicts the PDF of the extrapolated estimate

More details are available from Campbell et al. (2014). The formula (22) also can be written using the bivariate normal distribution (9).

\subsection{Confidence Interval of the Extrapolated Estimate}

Quantiles of distribution (22) can be used to evaluate the confidence interval of the extrapolated estimate. The profile log likelihood PLL method (Coles, 2001) adapted for the extrapolated estimate is another way to find the confidence interval (Campbell, et al., 2014). The $\log$ likelihood estimator (8) is expressed in terms of the extrapolated estimate $\hat{P}$ (19):

$$
l_{p}(\hat{\xi}, \hat{P})=-n \ln \left(\frac{\hat{\xi} \cdot c}{\hat{P}^{-\xi}-1}\right)-\left(1+\frac{1}{\hat{\xi}}\right) \sum_{i=1}^{n} \ln \left(1+\frac{z_{i}\left(\hat{P}^{-\xi}-1\right)}{c}\right)
$$

and maximized by the shape parameter:

$$
l_{m}\left(\hat{P}_{x}\right)=\underset{\xi}{\arg \max } l_{p}\left(\hat{\xi}, \hat{P}_{x}\right)
$$

and the extrapolated estimate:

$$
\hat{P}=\underset{\hat{P}_{x}}{\arg \max } l_{m}\left(\hat{P}_{x}\right)
$$

The difference between them, referred to as deviance statistic $D$ and is assumed to have $\chi^{2}$ distribution: 
The confidence interval includes the space where:

$$
D(p)<0.5 Q_{\chi^{2}}\left(P_{\beta}, d o f=1\right)
$$

$P_{\beta}$ is the confidence probability and $\mathrm{Q}_{\chi^{2}}$ is the quantile function of the $\chi^{2}$ distribution. The boundaries are found as the limits of $P_{x}$ that satisfy the condition (27), see Figure 6.

Glotzer, et al. (2016) systematically studied and compared different methods of calculating the confidence interval for the extrapolated estimate. The following methods were considered in details (initially standard delta method was also considered, but it has performed poorly and was excluded from the further study):

1. "Normal" method ( distribution 9)

2. "Lognormal" method (distribution 22)

3. "Boundary" method

4. Standard bootstrap method

5. Profile likelihood method (as briefly described above)

In addition to those five methods, three more techniques, termed indirect techniques, investigated were based on quantiles rather than extrapolated estimate for exceedance probability. Any of the methods mentioned above can be used to construct a confidence interval for the return level (the level to be exceeded with given probability $p$ ):

$$
x_{p}=\frac{\sigma}{\xi}\left(p^{-\xi}-1\right)
$$

The general scheme of calculations of the confidence interval through quantiles / return level is shown in Figure 7. The three methods adapted to the indirect approach using quantiles were:

6. Indirect "Lognormal" method

7. Indirect "Boundary" method

8. Indirect Profile likelihood method

A comparison study was performed on simulated data sampled from a GPD distribution. The performance was judged based on the percentage of cases where the confidence interval contained the true value (coverage). An accurate method should have the coverage close to the given confidence probability $\left(P_{\beta}=0.95\right)$. There were two series of calculations with 100 and 50 samples each.

The indirect profile likelihood method was shown to be the most accurate, see Glotzer, et al. (2016). The "normal", "log-normal", and indirect "log-normal" methods are slightly anticonservative (coverage $<95 \%$ ) with the log-normal method preferred among the three. The boundary method and indirect boundary methods are slightly conservative (coverage > 95\%). 
The bootstrap and profile likelihood methods performed poorly for negative and near-zero shape parameters.

\subsection{Convergence Study}

Campbell, et al. (2014) describes convergence study for the EPOT method, using the datasets from Smith et al. (2014) and Smith and Zuzick (2015). The results are shown in Figure 8.

The convergence test uses a moving average for 100 extrapolation data sets; the moving average is performed for the most probable value, mean value, and the upper boundary of the confidence interval calculated with the "log-normal" method. The most probable value is a better estimator when the sample volume is large, while the mean shows better (and more conservative) performance for smaller sample volumes. More details are available from Campbell, et al., (2014).

\subsection{On the Validation of EPOT}

A comprehensive validation study of the EPOT method is reported by Smith and Zuzick (2015). Motions of the ONR Tumblehome topsides (Bishop et al., 2005) configuration were simulated with a 3-DOF volume-based simulation (Weems and Wundrow, 2013; Weems and Belenky, 2015) for hundreds of thousands of hours to produce "true" values on rare exceedances (e.g. 30 degrees for roll or 11.5 degrees for pitch). Then small subsets (100 hrs each) was used by EPOT extrapolation. Smith and Zuzick (2015) used log-normal and boundary methods in carrying out their validation procedure for six relative wave headings considering roll, pitch, lateral and vertical accelerations. Using the same data set, Glotzer, et al. (2016) reported results for heave and pitch only for 30 and 45 degrees of heading, but used methods 2 through 9 for the confidence interval.

Total number of conditions reported by Smith and Zuzick (2015) was 23. The minimal acceptable coverage was set to $90 \%$. With log-normal confidence interval six conditions failed, while all the conditions are passed using the boundary method. These results could be expected as the log-normal method is slightly anti-conservative and the boundary method is slightly conservative, as mentioned above. Smith and Zuzick (2015) noted that all the conditions show acceptable results for restricted range of headings - aft of the beam seas. Similar conclusions were reached by Glotzer, et al. (2016).

\section{CURRENT STATUS AND FUTURE WORK}

The EPOT method has evolved significantly in the last three years. The main idea remains the same, however: extrapolate peaks over a threshold and use the envelope to ensure independence. The idea of a threshold was originally aimed to emphasize influence of nonlinearity. Now it also has a mathematical interpretation - it ensures the applicability of the second extreme value theorem. However, the relation between statistics and nonlinearity of ship motions is still a matter of current scientific interest. 


\section{ACKNOWLEDGEMENTS}

The work described in this paper has been funded by the Office of Naval Research, under Dr. Patrick Purtell, Dr. Ki-Han Kim and Dr. Thomas Fu. The authors greatly appreciate their support. The participation of Prof. Pipiras was facilitated by the Summer Faculty Program supported by ONR and NSWCCD under Dr. Jack Price (David Taylor Model Basin, NSWCCD). The authors would like to acknowledge fruitful discussions with Prof. Pol Spanos (Rice University), Dr. Art Reed, Mr. Tim Smith (David Taylor Model Basin, NSWCCD), and Prof. Ross Leadbetter (University of North Carolina ,Chapel Hill). 


\section{REFERENCES}

Belenky, V. and Campbell, B.L., 2012 "Statistical Extrapolation for Direct Stability Assessment", Proc. 11th Intl. Conf. on Stability of Ships and Ocean Vehicles STAB 2012, 243-256, Athens, Greece.

Belenky, V., Weems, K. and Lin, W.M., 2015 "Split-time Method for Estimation of Probability of Capsizing Caused by Pure Loss of Stability" in Proc. 12th Intl. Conf. on Stability of Ships and Ocean Vehicles STAB 2015, Glasgow, UK.

Bishop, R. C., W. Belknap, C. Turner, B. Simon, and Kim J. H., 2005 "Parametric Investigation on the Influence of GM, Roll damping, and Above-Water Form on the Roll Response of Model 5613.” Report NSWCCD-50-TR-2005/027.

Boos, D. D. and Stefanski, L. D., 2013 Essential Statistical Inference: Theory and Method. Springer.

Campbell, B. and Belenky, V., 2010 "Assessment of Short-Term Risk with Monte-Carlo Method" Proc. 11 th Int. Ship Stability Workshop, Wageningen, the Netherlands.

Campbell, B., Belenky, V. and Pipiras, V., 2014 "Properties of the Tail of Envelope Peaks and their Use for the Prediction of the Probability of Exceedance for Ship Motions in Irregular Waves" CSM7 Computational Stochastic Mechanics, Deodatis, G. and P. D. Spanos, eds. (accepted).

Campbell, B., Belenky, V. and Pipiras V., 2014a "On the Application of the Generalized Pareto Distribution for Statistical Extrapolation in the Assessment of Dynamic Stability in Irregular Waves" Proc. 14th Intl. Ship Stability Workshop, Kuala Lumpur, Malaysia, pp. 144-148.

Coles, S., 2001 An Introduction to Statistical Modeling of Extreme Values. Springer, London.

David, H. A. and Nagaraja, H. N., 2003. Order Statistics. Wiley Series in Probability and Statistics

Glotzer, D., Pipiras, V., Belenky, V., Campbell, B., Smith, T., 2016 "Confidence Intervals for Exceedance Probabilities with Application to Extreme Ship Motions", REVSTAT Statistical J. (Accepted)

IMO SLF 54/3/1 Development of Second Generation Intact Stability Criteria. Report of the Working Group at SLF 53 (part 2). Submitted by the Chairman of the Working Group, 2011, London, UK.

Kim, D.H., Belenky, V., Campbell, B. L. and Troesch, A. W., 2014 "Statistical Estimation of Extreme Roll in Head Seas" in Proc. of $33^{\text {rd }}$ Intl. Conf. on Ocean, Offshore and Arctic Engineering OMAE 2014, San-Francisco, USA.

Kobylinski, L. and Kastner, S. 2003 Stability and Safety of Ships. Regulation and Operation, Elsevier. 
McTaggart, K. A., 2000 "Ongoing Work Examining Capsize Risk of Intact Frigates Using Time Domain Simulation". In Contemporary Ideas of Ship Stability, D. Vassalos, et al. (eds), Elsevier Science, pp. 587-595.

McTaggart, K. A., de Kat J.O., 2000 "Capsize Risk of Intact Frigates in Irregular Seas”. Trans. SNAME, Vol. 108, pp 147-177.

Pickands, J., 1975 "Statistical Inference Using Extreme Order Statistics". The Annals of Statistics Vol. 3, No 1, pp. 119-131.

Reed, A., Beck, R. and Belknap, W., 2014 "Advances in Predictive Capability of Ship Dynamics in Waves", Invited Lecture at $30^{\text {th }}$ Symposium of Naval Hydrodynamics, Hobart, Australia.

Reiss, R.-D. and Thomas, M., 2007 Statistical Analysis of Extreme Values with Application to Insurance, Finance, Hydrology and Other Fields. 3rd Edition, Basel: Birkhäuser Verlag.

Smith, R. L., 1987 "Estimating tails of probability distributions." The Annals of Statistics Vol 15, pp.1174-1207.

Smith, T. C., 2014 "Example of Validation of Statistical Extrapolation Example of Validation of Statistical Extrapolation," Proc. of the 14th Intl. Ship Stability Workshop, Kuala Lumpur, Malaysia.

Smith, T. C., and Zuzick, A., 2015 "Validation of Statistical Extrapolation Methods for Large Motion Prediction" in Proc. 12th Intl. Conf. on Stability of Ships and Ocean Vehicles (STAB 2015), Glasgow, UK.

Weems, K., and Wundrow, D., 2013 "Hybrid Models for Fast Time-Domain Simulation of Stability Failures in Irregular Waves with Volume-Based Calculations for Froude-Krylov and Hydrostatic Forces", Proc. 13th Intl. Ship Stability Workshop, Brest, France.

Weems, K. and Belenky, V., 2015 "Fast Time-Domain Simulation in Irregular Waves With Volume-Based Calculations for Froude-Krylov and Hydrostatic Force" in Proc. 12th Intl. Conf. on Stability of Ships and Ocean Vehicles (STAB 2015), Glasgow, UK. 


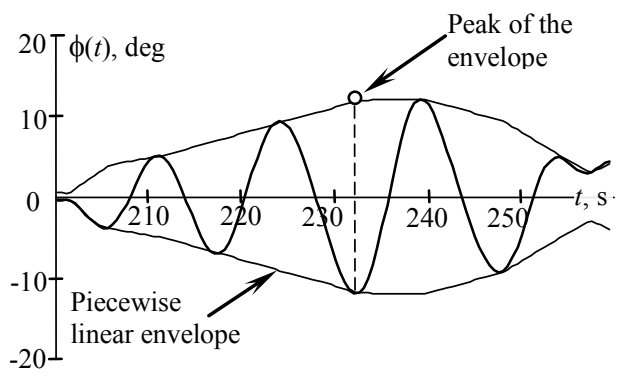

Figure 1: Piecewise linear envelope and its peak

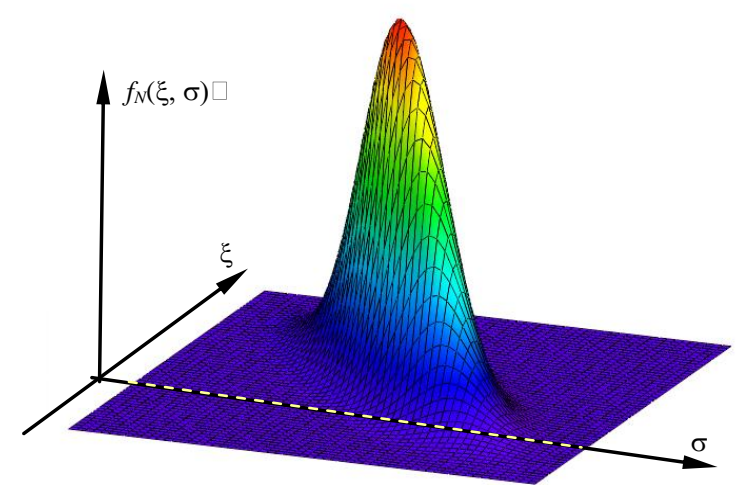

Figure 2: Joint distribution of GDP parameters

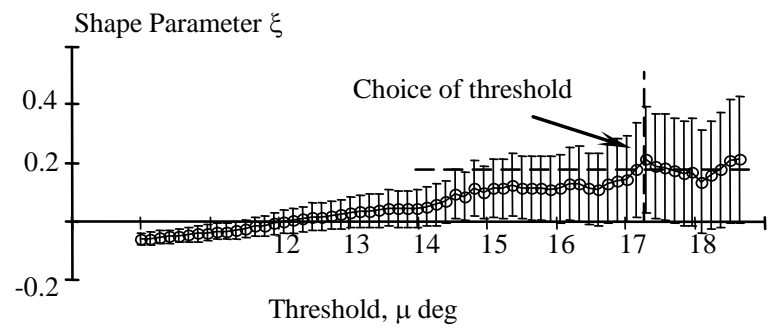

Figure 3 Choice of threshold by stabilization of the shape parameter

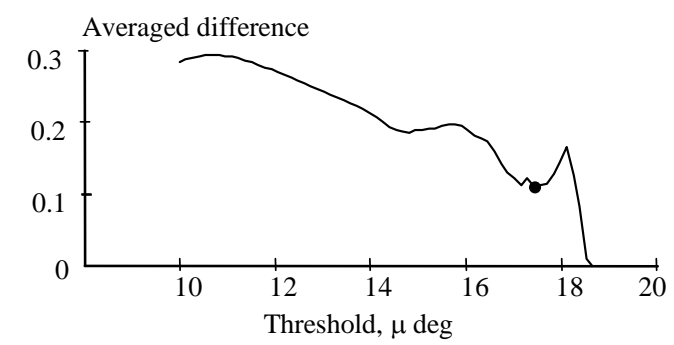

Figure 4 Choice of the Threshold based on the Global Minimum of the Equation (18) 


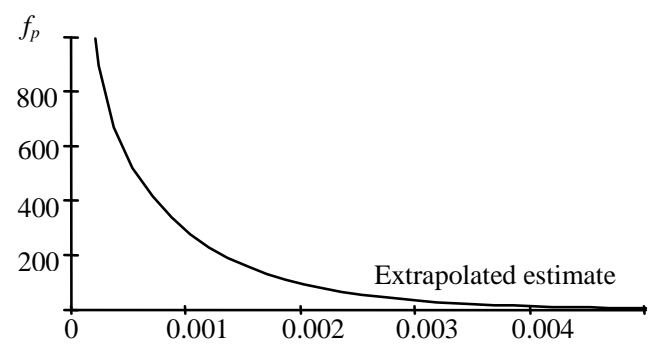

Figure 5: PDF of the Extrapolate Estimate (Campbell, et al., 2014).

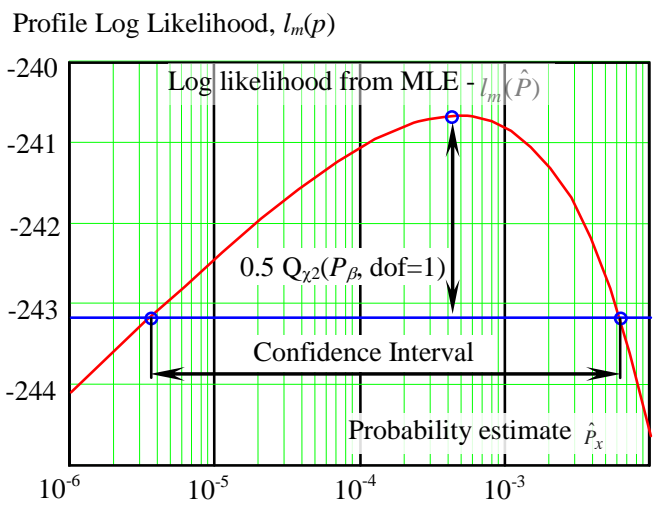

Figure 6. Profile Log Likelihood Method for Confidence Interval (Campbell, et al. 2014)

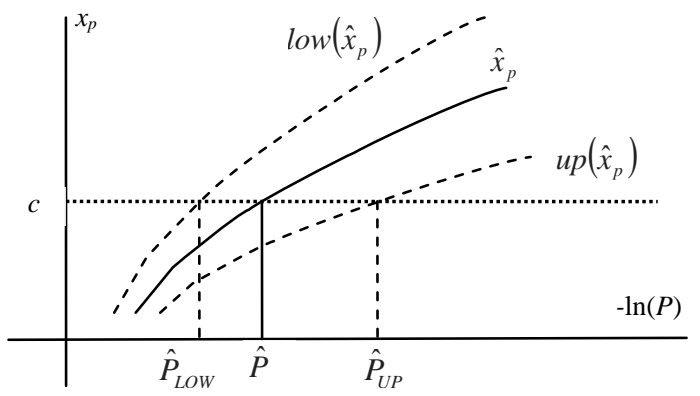

Figure 7 On Calculation of Confidence Interval using quantiles (Pipiras, et al., 2015)

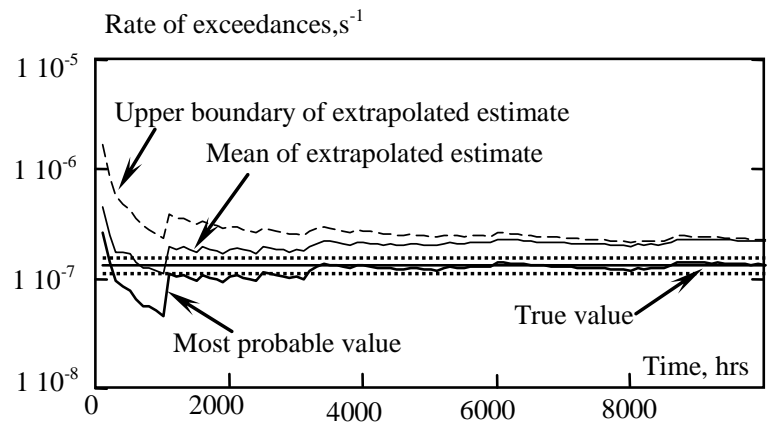

Figure 8 Convergence Test Using Moving Averages to Approximate Extrapolated Estimates (Campbell, et al., 2014) 